\title{
Simulation MANipulator BaSED ON STEP MOTORS FESTO
}

\author{
GANin, P. ; Kobrin, A.; Belousov, M. \& SHILIN, D.
}

Abstract: In this paper, we present modelling of the industrial manipulator with 5 degrees of freedom that using the software package Matlab Simulink. This model allows to evaluate the dynamic and static characteristics of the manipulator. The paper considers the possibility of solving the inverse kinematics using numerical methods. Also, in paper presented virtual environment that displays model motion in real time using a virtual 3-D model. As an example, in the paper shows applying the results of the work to the physical model of the manipulator. That model using the Festo stepper motors and the PLC Siemens. This work were performed in the Festo faculty of Moscow Power Engineering Institute.

Key words: manipulator, 3D modeling, inverse kinematics, PLC, step motor
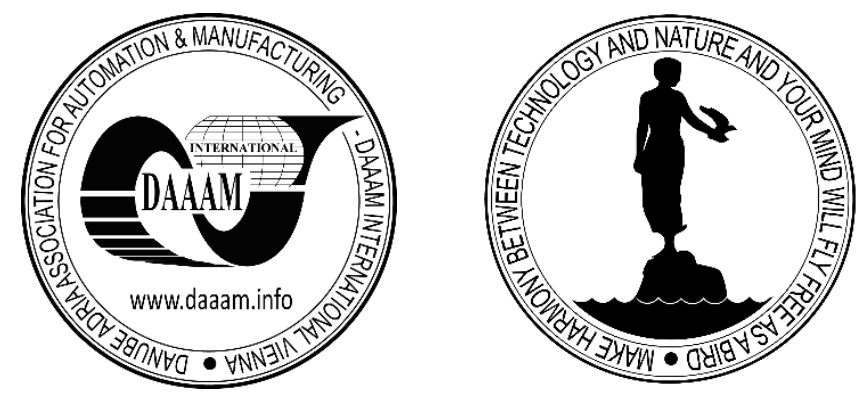

Authors' data: Dipl.-MSc. Ganin, P[avel]; Prof. Dr. Sc. Kobrin, A[leksandr]; Dipl.MSc. Belousov, M[aksim]; Dipl.-MSc. Shilin, D[enis]; Moscow Power Engineering Institute (National Research University), Krasnokazarmennaya 14, 111250, Moscow, Russia, GaninPY@mpei.ru, KobrinAI@yandex.ru, BelousovMI90@gmail.com, deninfo@mail.ru.

This Publication has to be referred as: Ganin, P[avel]; Kobrin, A[lexandr]; Belousov, M[aksim] \& Shilin, D[enis] (2015). Simulation Manipulator Based on Step Motors Festo, Chapter 11 in DAAAM International Scientific Book 2015, pp.127-134, B. Katalinic (Ed.), Published by DAAAM International, ISBN 978-3-902734-05-1, ISSN 1726-9687, Vienna, Austria

DOI: $10.2507 /$ daaam.scibook.2015.11 
Ganin, P.; Kobrin, A.; Belousov, M. \& Shilin, D.: Simulation Manipulator Based o...

\section{Introduction}

Currently manipulators are widely used in various fields of human activity, including industry. In this paper, shows modeling an industrial manipulator based on step motors of firm Festo, using the programmable logic controller (PLC) Siemens as a control device. Design process of the manipulator consists of various tasks that require detailed consideration.

\section{Construction and mechanical features of the manipulator}

The first important task is to determine the structure and mechanics of the manipulator. Mechanical structure of the future model (Fig. 1) is used to indicate the mobility of the links and the geometrical parameters of the design.

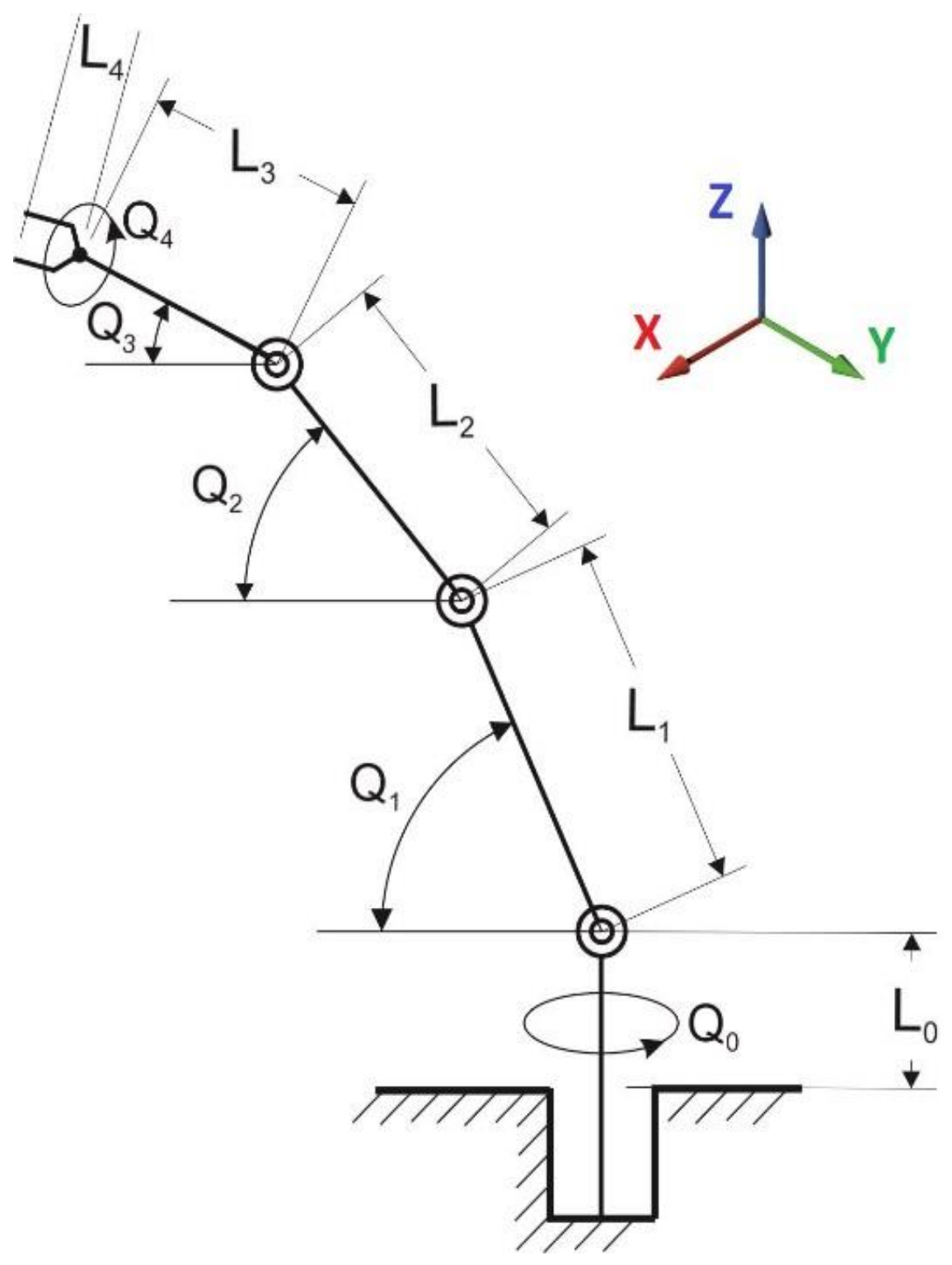

Fig. 1. Mechanical scheme of the manipulator 
Fig. 1 uses the following conventions: the height of the base $L_{0}=200[\mathrm{~mm}]$; the length of the 1st, 2nd and 3rd links, respectively $L_{1}=600, L_{2}=500, L_{3}=400[\mathrm{~mm}]$; the length of gripper $L_{4}=100[\mathrm{~mm}] ; Q_{0}$ - rotation around the axis Z; $Q_{1}, Q_{2}, Q_{3}$ - the slope of the first, second and third links, respectively, to the XY plane of the axis Z; $Q_{4}$ - gripper rotation around the axis $\mathrm{Z}$. The above design is a manipulator with 5 degrees of freedom. At angles impose the following restrictions: $-180 \leq Q_{0,4} \leq 180\left[^{\circ}\right]$; $0 \leq Q_{1} \leq 45\left[^{\circ}\right] ; 0 \leq Q_{2,3} \leq 90\left[^{\circ}\right]$. To determine the gripper coordinates in the space we should solve the forward kinematics problem of the manipulator. We neglect the manipulator rotation and fix an angle $Q_{4}$. Proceeding from the geometric constraints, we compose the following system of equations:

$$
\begin{gathered}
x=L_{1} \cdot \sin Q_{1} \cdot \cos Q_{0}+L_{2} \cdot \sin \left(Q_{1}+Q_{2}\right) \cdot \cos Q_{0}+\left(L_{3}+L_{4}\right) \cdot \\
\cdot \sin \left(Q_{1}+Q_{2}+Q_{3}\right) \cdot \cos Q_{0} \\
y=L_{1} \cdot \sin Q_{1} \cdot \sin Q_{0}+L_{2} \cdot \sin \left(Q_{1}+Q_{2}\right) \cdot \sin Q_{0}+ \\
+\left(L_{3}+L_{4}\right) \cdot \sin \left(Q_{1}+Q_{2}+Q_{3}\right) \cdot \sin Q_{0} \\
z=L_{0}+L_{1} \cdot \cos Q_{1}+L_{2} \cdot \cos \left(Q_{1}+Q_{2}\right)+\left(L_{3}+L_{4}\right) \cdot \cos \left(Q_{1}+Q_{2}+Q_{3}\right)
\end{gathered}
$$

Attainability domain of manipulator (working area) (Fig. 2) can be constructed by using the system of equations (1).

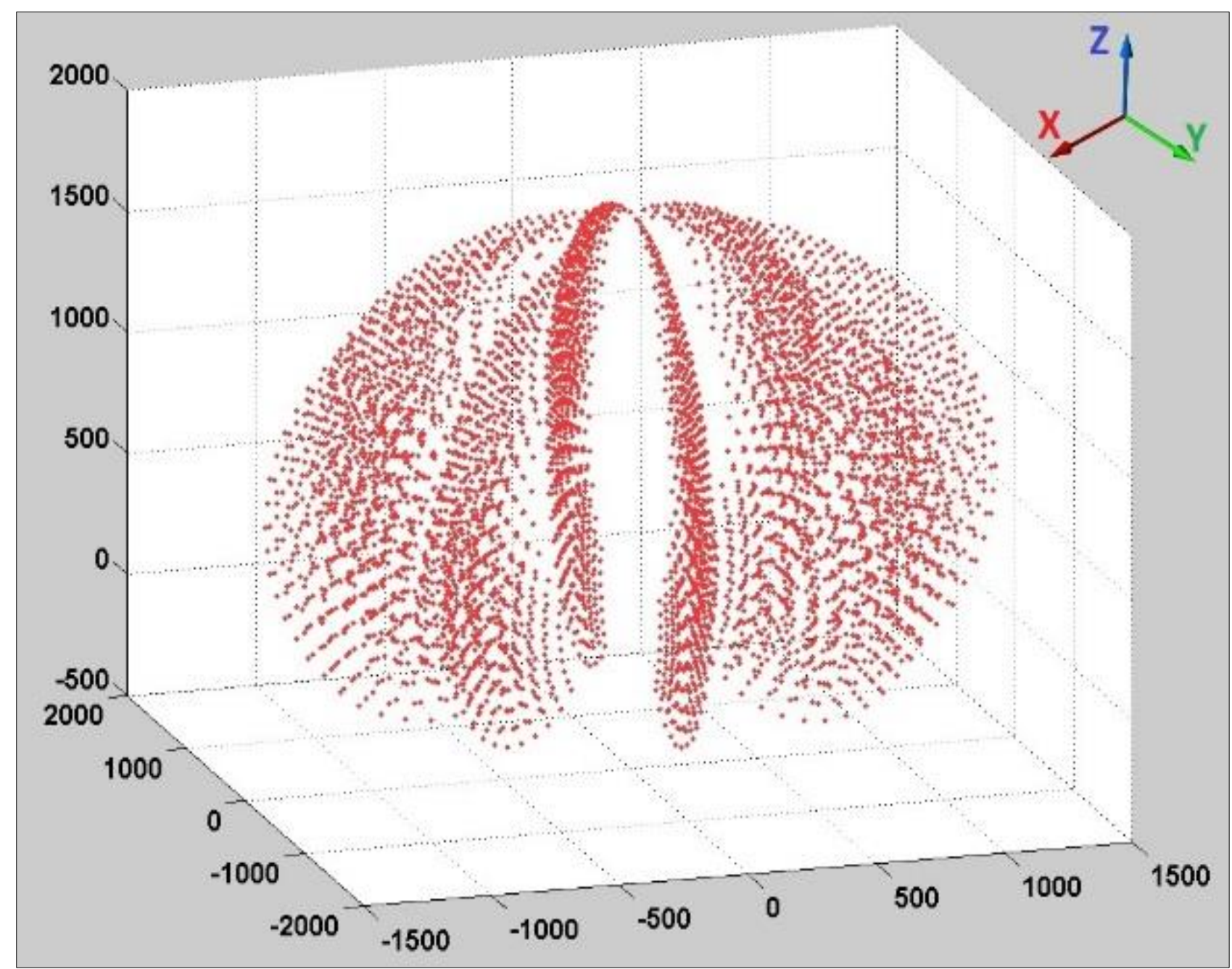

Fig. 2. The attainability domain in the space 
Ganin, P.; Kobrin, A.; Belousov, M. \& Shilin, D.: Simulation Manipulator Based o...

In fact, the working area in the space is a rotation body of attainability domain on the plane (Fig. 3) around the axis Z.

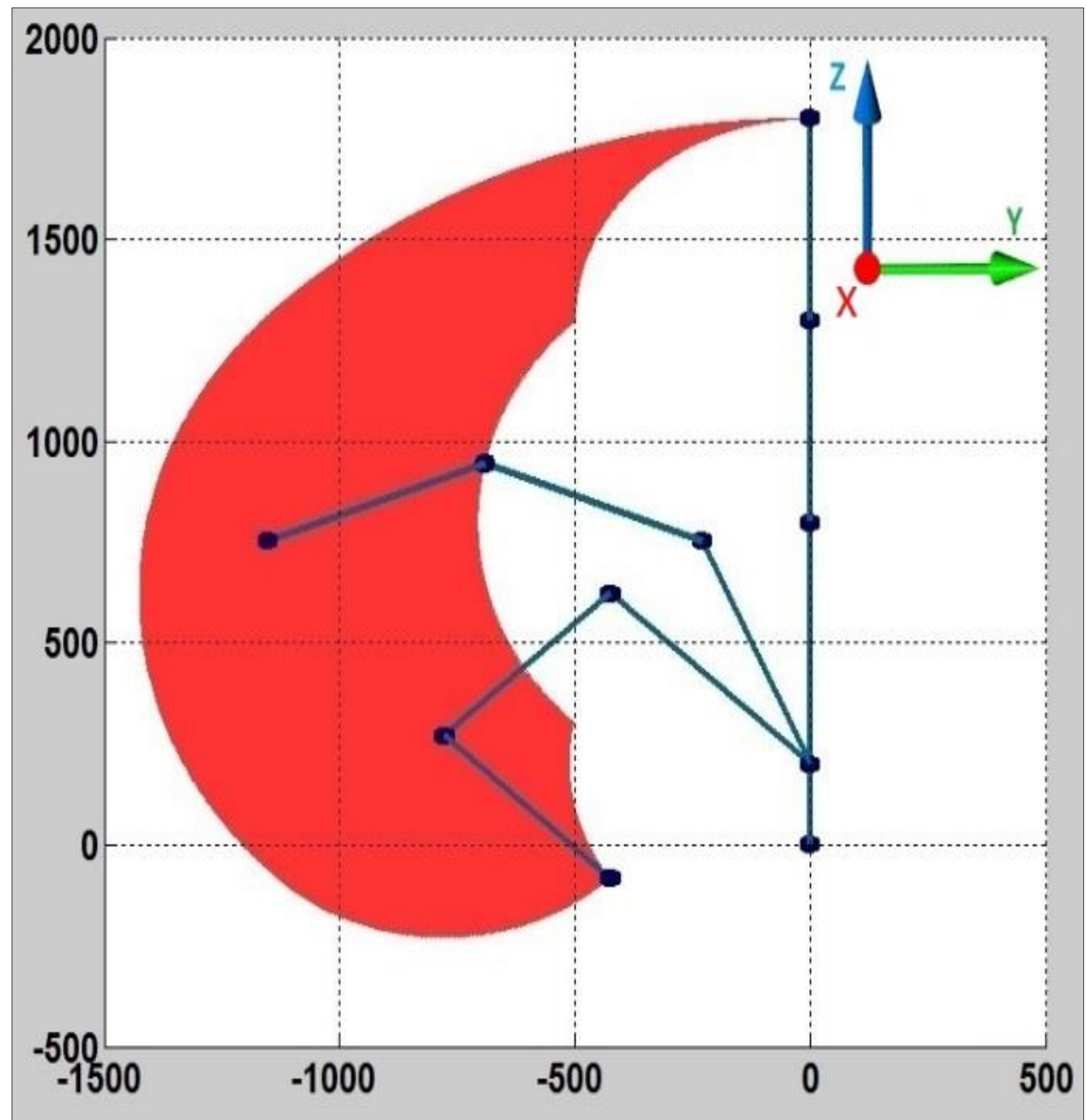

Fig. 3. The attainability domain on the plane

Also Fig. 3 shows the extreme and average position of the manipulator. To orient the arm at a specific point we should determine the angles for which turn the links, but since there are more unknowns than equations in the system, it is necessary to add the following equation:

$$
Q_{0}=\operatorname{arctg}\left(\frac{y}{x}\right)
$$


Equation (2) was derived from the geometry of the manipulator. To solve the inverse kinematics (location coordinates) we should solve the system of four equations (1) and (2). Analytical solution of this system is not possible, so we should use numerical methods. The most popular is an iterative method of Newton - Raphson.

\section{The equipment included in the model}

Next step is selection the components that meet the requirements of the task. Fig. 4 shows a diagram of the manipulator, which includes the major functional components and scheme of data exchange.

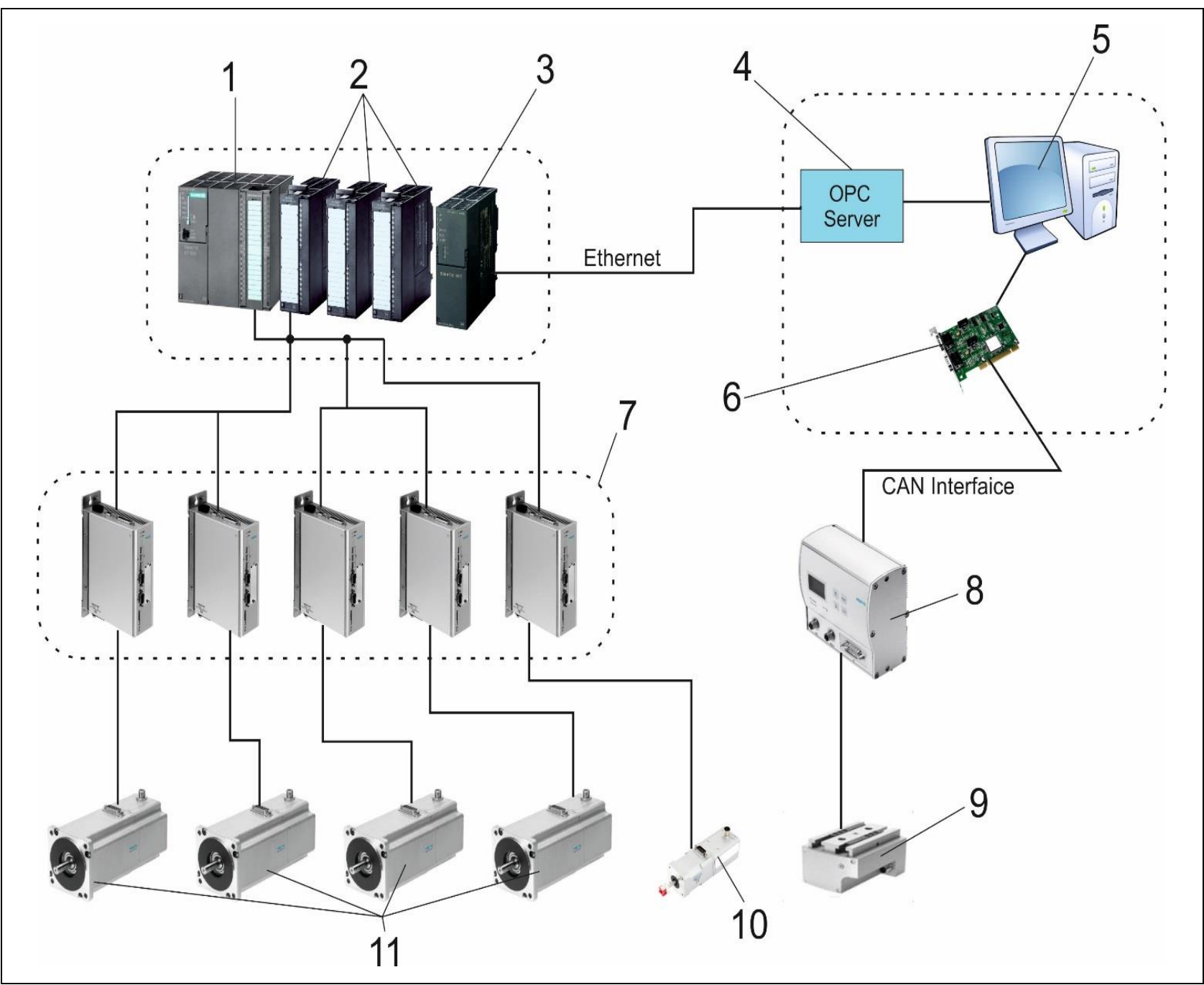

Fig. 4. Functional diagram of the manipulator

In Fig. 4 you can see: 1 - programmable logic controller (PLC) Siemens CPU313C-2 DP; 2 - analogue extension module SM334; 3 - communication module CP 343-1 Lean; 4 - OPC-Server (Object Linking and Embedding for Process Control); 5 - personal computer (PC), 6 - extension board of CAN-interface for PC; 7 - motor controllers Festo CMMS-ST-C8-7-G2; 8 - controller of gripper Festo SFC-DC-VC-3E-H2- CO; 9 - gripper Festo HGPLE-25-40-2; 10 - step motor Festo EMMS-ST-42-SSE-G2; 11 - step motors Festo EMMS-ST-87-L-SE-G2. 
Ganin, P.; Kobrin, A.; Belousov, M. \& Shilin, D.: Simulation Manipulator Based o...

The controller Festo that receives data from the PC is used to open / close the gripper. The motor (10) allows rotation of the gripper and the motors (11) set in motion the manipulator and tilt links. Low-level controllers Festo perform the control of motors. Their control is carried out by high-level controller Siemens, which receives data from the communication module via Ethernet from the OPC-server. Using the PC as a computing center improves system performance and increases the speed of the coordinate's calculation. PC transmits the data via OPC-server to the controller Siemens, where it adjust the direct change in the coordinates. Positioning (changing of coordinates) with the use of Siemens can be implemented by using a variety of controls (P, PI, PID controls). It provides the ability to customize the speed and accuracy of the system.

\section{Virtual reality model of manipulator}

Subsequent task of the manipulator design is to create a virtual model of the system. Software Matlab Simulink is used for this purpose. By using the Simulink SimMechanics we construct model of the manipulator, which includes elements that represent the work of the mechanics of links and joints of the manipulator. The block model of virtual reality Simulink VR Sink is used to visualize the motion of the manipulator. The 3-D model of the manipulator for this unit is created in advance (e.g. in software product Kompas 3D). The motion of the model is the result of the data transmission from the model SimMechanics VR Sink. Access to the virtual model (Fig. 5 ) is possible from the Internet.

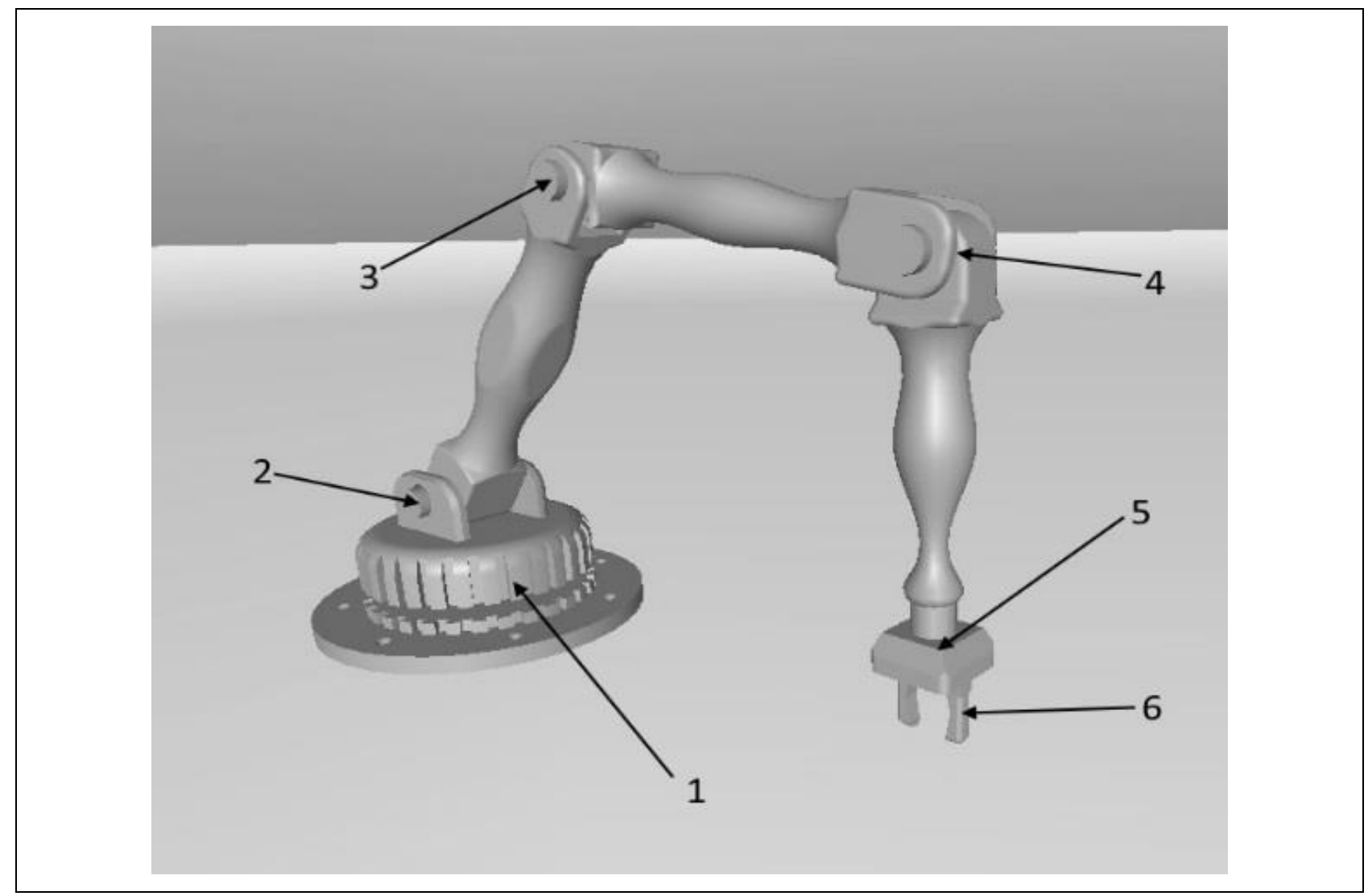

Fig. 5. Virtual model of the manipulator 
Rotation of the base 1 (Fig. 5) and rotation of links 2-4 (Fig. 5) are performed by a motor 11 (Fig. 4). Rotation of the body gripper 5 (Fig. 5) is realized by a motor 10 (Fig. 4). The working body of the manipulator is gripper 6 (Fig. 5).

\section{Virtual reality model of manipulator}

This model of the manipulator was physically modelled with using the Festo equipment. Fig. 6 shows the power supply for the system, controllers Festo and the PLC Siemens. Fig. 7 shows the engines and gripper Festo.

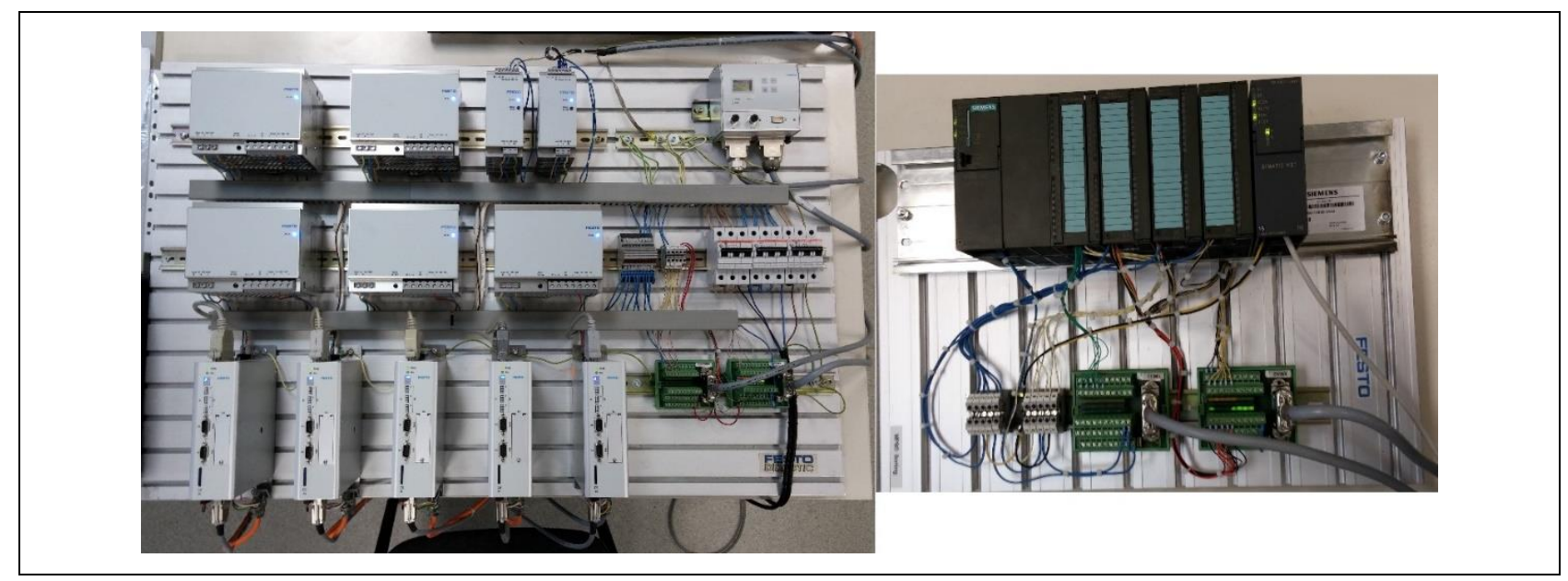

Fig. 6. The power supply system and the controllers

The entire system is placed in three distinct mounting plate, that making possible to separate the control feeding and executive parts.

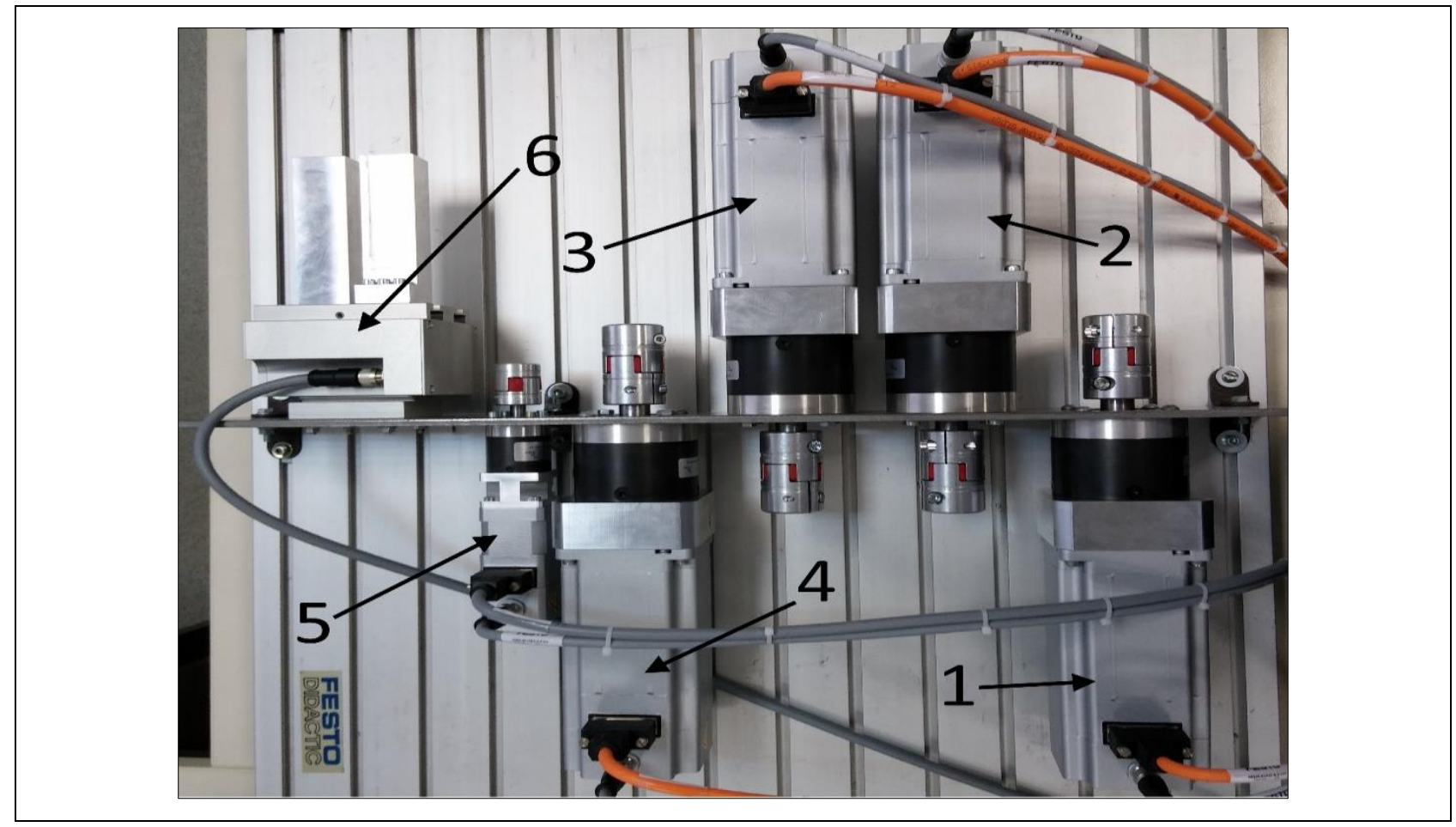

Fig. 7. The step motors and the gripper 
Ganin, P.; Kobrin, A.; Belousov, M. \& Shilin, D.: Simulation Manipulator Based o...

The equipment on Fig. 7 performs the following functions of the manipulator: base rotation - by the motor 1 ; links rotation - by the motors 2,3 , 4; working body of the manipulator is gripper 6 , the rotation of which carries by the motor 5 . All motors equipped with gearboxes that reduce the speed of rotation.

\section{Conclusion}

The paper shows the possibility of visualizing for the motion simulation of the manipulator with using industrial equipment.This model of an industrial manipulator has several advantages due to the use of step motors that provide sufficient accuracy and reliability. They also have the ability to fine-tune (for example, to stop at the moment of a sharp increase in resistance). The results can be interpreted to solve similar problems.

\section{References}

Amosov A.A., Dubinsky Y.A., Kopchenova N.V. (1994) Computational Methods for EngineersTextbook for higher educational institutions. M.: Higher School

Kaganov Y.T., Karpenko A.P. Mathematical modeling of the kinematics and dynamics of the robot manipulator of the "trunk". "Science and Education" - electronic scientific and technical publications, Available from: http://technomag.bmstu.ru/doc/133731.html Accessed: 2015-02-11

Musalimov V.M., Zamoruev G.B., Kalapyshina I.I., Perechesova A.D., Nuzhdin K.A. (2013). Modeling of mechatronic systems in the environment MATLAB (Simulink / SimMechanics): Textbook for higher educational institutions. St. Petersburg: ITMO. Solovyov S.G. Creating an object VRWorld. Matlab.Exponenta, Available from: http://matlab.exponenta.ru/virtualreality/interface.php Accessed: 2015-02-11

The MathWorks Inc. Modeling Inverse Kinematics in a Robotic Arm, Available from: http://www.mathworks.com/help/fuzzy/examples/modeling-inverse-kinematics-in-arobotic-arm.html Accessed: 2015-02-11 